\title{
Sex-Related Hemispheric Lateralization of Amygdala Function in Emotionally Influenced Memory: An fMRI Investigation
}

\author{
Larry Cahill, ${ }^{1,4}$ Melina Uncapher, ${ }^{1}$ Lisa Kilpatrick, ${ }^{1}$ Mike T. Alkire, ${ }^{2}$ and Jessica Turner ${ }^{3}$ \\ ${ }^{1}$ Center for the Neurobiology of Learning and Memory and Department of Neurobiology and Behavior, ${ }^{2}$ Department of \\ Anesthesiology, and ${ }^{3}$ Department of Psychiatry and Human Behavior, University of California, \\ Irvine, California, 92697-3800, USA
}

\begin{abstract}
The amygdala appears necessary for enhanced long-term memory associated with emotionally arousing events. Recent brain imaging investigations support this view and indicate a sex-related hemispheric lateralization exists in the amygdala relationship to memory for emotional material. This study confirms and further explores this finding. Healthy men and women underwent functional Magnetic Resonance Imaging (fMRI) while viewing a series of standardized slides that were rated by the subjects as ranging from emotionally neutral to highly arousing. Two weeks later, memory for the slides was assessed in an incidental recognition test. The results demonstrate a significantly stronger relationship in men than in women between activity of the right hemisphere amygdala and memory for those slides judged as arousing, and a significantly stronger relationship in women than in men between activity of the left hemisphere amygdala and memory for arousing slides. An ANOVA confirmed a significant interaction between sex and hemisphere regarding amygdala function in memory. These results provide the strongest evidence to date of a sex-related hemispheric lateralization of amygdala function in memory for emotional material. Furthermore, they underscore the view that investigations of neural mechanisms underlying emotionally influenced memory must anticipate, and begin to account for, the apparently substantial influence of sex.
\end{abstract}

The amygdala appears crucially involved with enhanced memory associated with emotionally arousing events in both animal (McGaugh 2000) and human (Cahill 2000) subject studies. For example, activity of the human amygdala during encoding of arousing material relates significantly to long-term memory of that material but not to memory of nonarousing material (Cahill et al. 1996, 2001; Canli et al. 1999, 2000, 2002; Hamann et al. 1999). Conversely, bilateral amygdala lesions reduce or abolish enhanced long-term memory associated with emotional arousal, although they do not affect memory for relatively neutral material or affect emotional reactions per se to arousing stimuli (Cahill et al. 2000).

More recently, human brain imaging evidence has begun to reveal a sex-related hemispheric lateralization of amygdala function with respect to memory for emotionally arousing material. For example, in both a glucose PET investigation (Cahill et al. 2001) and an fMRI investigation (Canli et al. 2002), activity of the right, but not left, hemisphere amygdala related significantly to long-term incidental memory for arousing material in men but not in women, whereas activity of the left, but not right, hemisphere amygdala related significantly to memory for arousing material in women but not in men. As noted recently by Pizzagalli and colleagues (2003), such claims about lateralized amygdala function "require systematic replication." Providing such a replication was the first major aim of the present study.

Evidence for hemispheric lateralization of brain function derived from brain imaging investigations must be approached with caution, as thresholding differences may create the appearance of asymmetrical function greater than that which actually

\section{${ }^{4}$ Corresponding author.}

E-MAIL Ifcahill@uci.edu; FAX (949) 824-5244.

Article and publication are at http://www.learnmem.org/cgi/doi/10.1101/ Im.70504. exists. One method to further substantiate the existence of a sex-related hemispheric lateralization of amygdala function with respect to memory for arousing material is to demonstrate the existence of a sex-by-hemisphere interaction in this relationship (Pizzagalli et al. 2003), which no previous study of the amygdala sex-related lateralization in relation to memory has reported. This issue is perhaps of even greater importance in considering regions, such as the amygdala, that are prone to susceptibility artifacts and reduced signal-to-noise ratio with functional Magnetic Resonance Imaging (fMRI) (LaBar et al. 2001). Thus, a second major aim of the present study was to document the existence of a sex-by-hemisphere interaction in amygdala function in emotionally influenced memory. The study procedures were patterned after an earlier fMRI investigation of amygdala function in emotionally influenced memory (Canli et al. 2000).

\section{RESULTS}

\section{Behavioral Data}

Sex-related differences in the arousal ratings of the pictures were consistent with those found by Canli et al. (2002). Specifically, women rated more pictures as most highly arousing (rating of four) than did men $(\mathrm{t}(21)=-1.82, P=0.04)$. And similar to the findings of Canli et al. (2002), there were no sex-related differences in the number of false recollections $(\mathrm{F}(1,21)=0.47$, $P>0.05 ; 19 \%$ for men and $16 \%$ for women). Also, significant sex-related differences in memory performance existed only for pictures rated as most highly arousing. However, unlike the study of Canli et al. (2002), in the present study men exhibited better retention than did women for the most arousing pictures. Specifically, men recalled a higher percentage of pictures rated as most highly arousing $(\mathrm{t}(21)=2.04, P=0.05$; Fig. 1 A) than did women. Men also remembered with certainty a higher percentage of pictures rated most highly arousing $(\mathrm{t}(21)=2.07, P=0.05$; 
A)

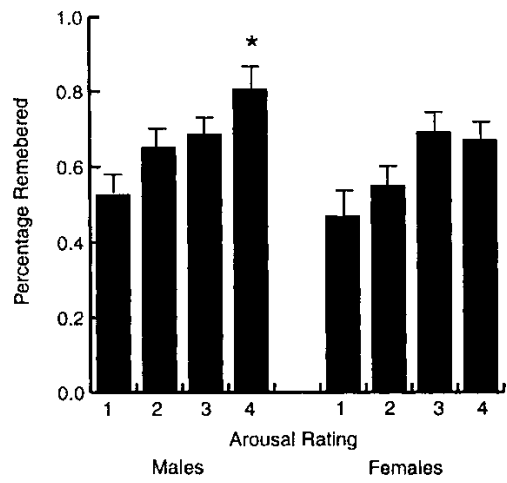

B)

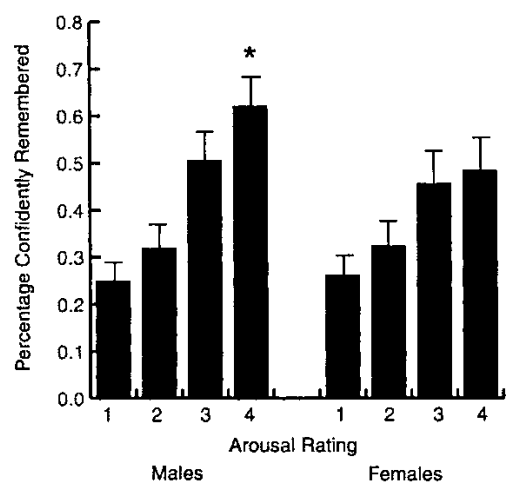

Figure 1 Results of the memory testing. Males exhibited significantly higher retention than did females of slides rated as most highly arousing (rated four) both in terms of total recall $(A)$ and in terms of recall with total confidence $(B) .{ }^{*} P<0.05$ from the corresponding value in females.

Fig. 1B.) Both sexes remembered a higher percentage of pictures rated as highly arousing than a percentage of pictures rated as not arousing at all $(\mathrm{t}(20)=-3.05, P=0.01$ for women; $\mathrm{t}(22)=-3.81, P=0.001$ for men).

\section{Amygdala Function}

The results of the between-groups, random-effects analysis comparing women and men are shown in Figure 2. In women, the left hemisphere amygdala, but not the right, demonstrated greater blood oxygen level-dependent (BOLD) signal with better memory performance and increasing arousal ratings than occurred in men. In contrast, in men the right hemisphere amygdala, but not the left, demonstrated greater BOLD signal with better memory performance and increasing arousal ratings than occurred in women. In addition, the significant amygdala activation was more medial in women than it was in men, with the peak activation located $4 \mathrm{~mm}$ more medially in women than in men.

Analysis of amygdala activity independently in men and women revealed significant activation clusters in the right but not left hemisphere amygdala in men, and in the left but not right hemisphere amygdala in women $(P<0.001$, uncorrected for multiple comparisons). These results are shown in Figure 3A. The results of the sex-by-hemisphere ANOVA on the parameter estimates of amygdala activity revealed a significant interaction between sex and hemisphere $(\mathrm{F}(1,13)=7.99, P=0.022$; Fig. 3B). The additional ANOVA using parameter estimates from amygdala voxel coordinates derived from an independent study of amygdala participation in emotionally influenced memory
(Canli et al. 2002) also revealed a significant sex-by-hemisphere interaction $(\mathrm{F}(1,13)=6.35, P=0.026)$.

Although not central to the primary amygdala question at issue in this study, the within-groups analysis also revealed other brain regions demonstrating significantly greater BOLD signal with both better subsequent memory and increasing arousal ratings. All such significant regions with a minimum voxel cluster size of four voxels are listed in Table 1 . Men showed significant $(P<0.001)$ activations including the right anterior hippocampus, right globus pallidus, bilateral lateral parietal, and right frontal cortex. Four out of six significant activations in men were located in the right hemisphere. In contrast, all significant activations detected in women were located in the left hemisphere, including the left posterior cingulate, left middle temporal gyrus, and left inferior parietal cortex.

\section{DISCUSSION}

The results of this study by use of event-related fMRI strongly confirm the sex-related hemispheric lateralization of amygdala function in memory for emotionally arousing material originally reported in a glucose PET study (Cahill et al. 2001). For the left hemisphere amygdala, a significantly stronger relationship between its activity at encoding and long-term memory for arousing pictures was detected in women relative to men, although no such difference occurred in the right hemisphere amygdala. Conversely, for the right hemisphere amygdala, a significantly stronger relationship between its activity at encoding and long-term memory for arousing pictures was detected in men relative to women, although no such difference occurred in the left hemisphere amygdala. A second analysis independently assessing the group activity of the amygdala in men and women confirmed this result, including documentation of a significant sex-byhemisphere interaction in the amygdala relationship to memory for arousing pictures. These results provide the most compelling demonstration to date of a sex-related hemispheric lateralization of amygdala function in memory for emotionally arousing events and, together with previous studies (Cahill et al. 1996, 2001; Canli et al. 1999, 2000, 2002; Hamann et al. 1999), leave no reasonable doubt about the existence of this sex-related laterality in humans, at least as regards negatively valenced material.

Several points concerning this lateralization should be emphasized. First, the consistent amygdala lateralization seen to date has been in its relationship to long-term memory for emotionally arousing events, as opposed to its relation to emotional reactions per se. Canli et al. (2002), for example, found that activity of the left amygdala related to emotional reactions to pictures in both men and women, despite that fact that they also found a very similar sex-related hemispheric lateralization of amygdala function with respect to memory as reported here. Second, we emphasize that our findings do not indicate that the left hemisphere amygdala in men and the right hemisphere amygdala in women have no function(s) in memory. They indicate only that there is a significant lateralization of these functions, at least with respect to the type of information learned in this experiment. Third, and related to the second point, we emphasize that all experiments to date in which the sex-related lateralization was found, including this experiment, involved emotionally negative material. Although we argue that arousal, rather than valence, is the key to amygdala engagement in memory processes (Cahill and McGaugh 1990; Cahill 2000), it may be that an identical sex-related lateralization of amygdala function will not exist in similarly arousing but emotionally positive learning situations.

Although the evidence to date points compellingly to the existence of a sex-related hemispheric lateralization of amygdala function in relation to memory for emotional events, it does not 


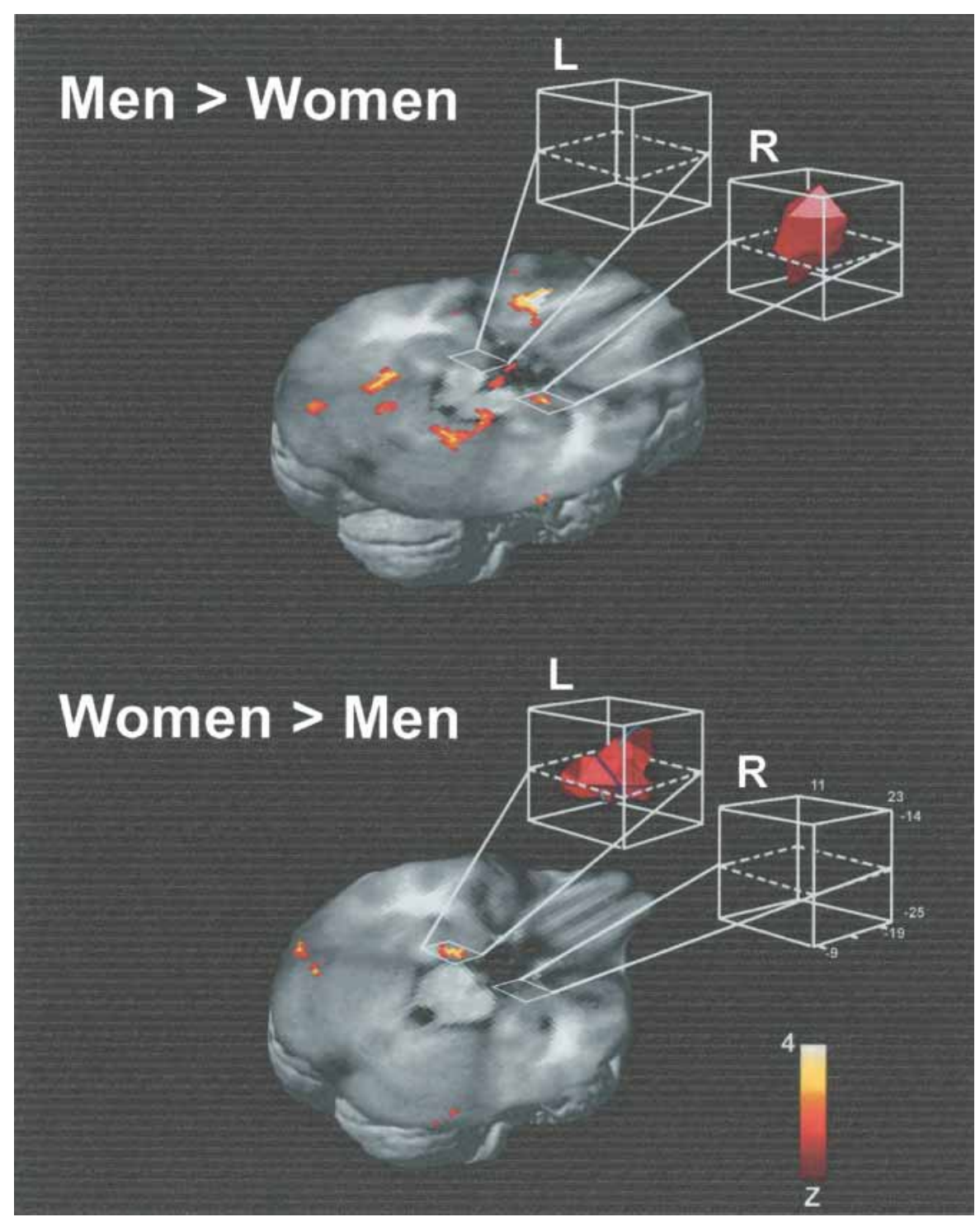

Figure 2 The results of the random effects, between groups' analysis comparing women and men, thresholded at $P<0.0025$. White boxes highlight the activity in the amygdala. $L$ indicates left hemisphere; R, right hemisphere. Talairach coordinates for the peak voxel in each cluster were 20, -10 , -11 for the men $>$ women effect; $-16,-14,-16$ for the women $>$ men effect.

yet clarify what this lateralization means and what combination of biological (nature) and psychological (nurture) factors produced it. Answering these questions is now crucial for future investigation. One hypothesis we have pursued in this regard concerns hemispheric specialization in the processing of relatively global holistic aspects of a stimulus or scene versus processing of relative local fine-detailed aspects of the stimulus or scene. Substantial evidence points to a bias of the right hemisphere in processing global information and to a bias of the left hemisphere in processing local information (see Delis et al. 1986; Fink et al. 1997; Ivry and Robertson 1998). We combined this fact with the sex-related hemispheric specialization of the amygdala ("males right/females left") to detect a sex-related difference in the impairing effect of a drug that presumably impairs amygdala function in memory, the $\beta$-adrenergic antagonist propranolol (Cahill and van Stegeren 2003). It appears that couching our understanding of amygdala function in terms of the hemisphere in which each amygdala operates is one method to begin to un- derstand the functional significance of the sex-related amygdala lateralization in memory.

The "memory modulation" hypothesis of amygdala function (McGaugh 2000) requires that the amygdala work in concert with other brain regions to influence memory. Although the focus of this article is the amygdala, our analyses revealed other brain regions with activity that is similarly related to memory (Table 1). Interestingly, six out of eight significant activations in men were located in the right hemisphere. In contrast, all significant activations detected in women were located in the left hemisphere. It is also of interest that right hemisphere hippocampal and frontal regions in men showed activation patterns similar to those of the right amygdala. A recent study from our laboratory (Kilpatrick and Cahill 2003) provided the first evidence of heightened outflow from the amygdala to other brain regions in emotional compared with neutral learning conditions, most notably from the right hemisphere amygdala in men to ipsilateral frontal and parahippocampal regions.

A steadily growing number of studies indicate sex-related influences on amygdala function. For example, Killgore and colleagues (2001) report sexrelated differences in amygdala responsiveness to faces, whereas an extensive series of studies by the Gurs and their colleagues (Gur et al. 2002) documents sex-related differences in many aspects of brain function, including the size of the amygdala relative to other brain regions. Similarly Giedd and colleagues (1997) found a sex-related difference in the relative sizes of the amygdala and hippocampus. The amygdala is larger relative to total cerebral size in men compared with women, and in boys compared with girls (Durston et al. 2001; Goldstein et al. 2001). Zubieta and colleagues (1999) found significant sex-related differences in opioid receptor binding within the amygdale. A recent meta-analysis of the literature by Wager et al. (2003) identified a female-left/maleright lateralization of an "extended amygdala" area in relation to emotional arousal. And recently, Toufexis and Davis (2002) reported a pronounced influence both of sex and of the sex hormone progesterone on an amygdala-dependent process-fear potentiated startle in rats. Finally, intravenous administration of procaine, which can produce a dramatic emotional response, produced significantly greater left amygdala activation in women compared with men (Adinoff et al. 2003). The issue of sex-related influences may also inform other views of amygdala functional hemispheric specialization (Morris et al. 1998; Buchanan et al. 2001; Phelps et al. 2001), none of which to date have accounted for potential influences of sex.

It is possible that the sex-related differences in recognition memory performance seen in the present study and the one by Canli et al. $(2000,2002)$ are at least partially related to sex-related 
A
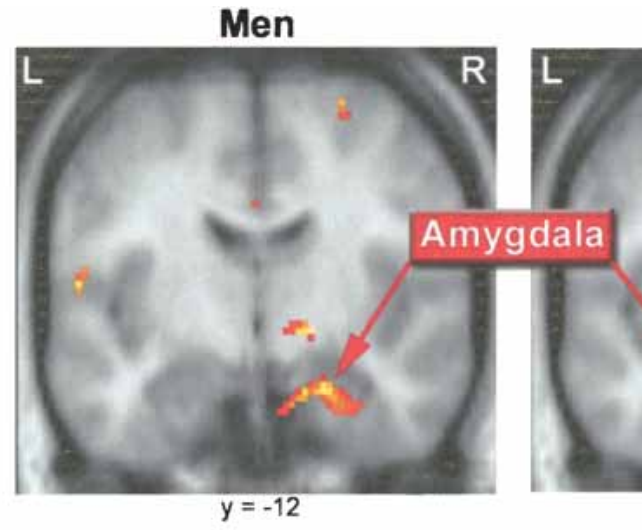

Women
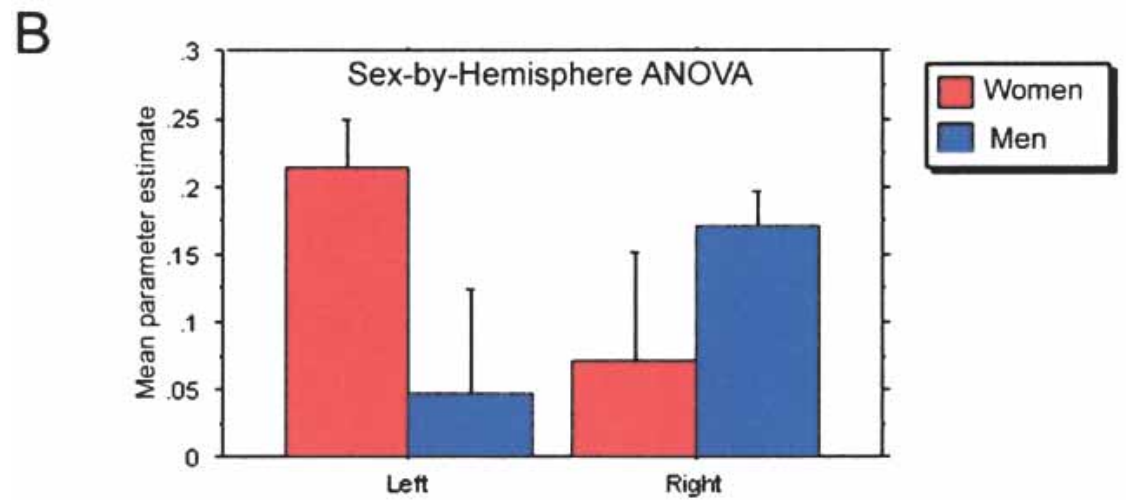

Figure 3 (A) Results of the random effects, within groups analysis of amygdala activity in males and females, thresholded at $P<0.005$. (B) Mean parameter estimate of amygdala activity in the left and right hemispheres, in males and in females. There was a significant interaction between sex and hemisphere in amygdala function by this measure (see text for additional details). Talairach coordinates for the peak voxel in each cluster were $22,-12,-15$ for the effect in men, and $-18,-12,-15$ for the effect in women.

differences in response bias. For example, the current data cannot eliminate the possibility that the male's higher hit rate for highly arousing pictures was due in part to an increased bias to respond "old" to any emotionally arousing picture (foils as well as targets). Measures such as $\mathrm{d}^{\prime}$ are designed to account for such differences in response biases by considering a subject's false alarm rate as well as their hit rate. $\mathrm{d}^{\prime}$ could not be computed in the current study as the lack of arousal ratings for the foils rendered it impossible to determine the subject's false alarm rate for each arousal level. Future studies should include subject ratings for the foils used on recognition tests. Although the present study cannot rule out the influence of response bias, the sexrelated lateralization of amygdala function reported here is entirely consistent with that of our earlier investigation using free recall tests (Cahill et al. 2001), in which response bias could not account for the findings.

In conclusion, the present findings provide the most compelling demonstration to date of a sex-related hemispheric lateralization of amygdala function with respect to memory for emotionally arousing events. As such, they strengthen the argument that a complete understanding of neurobiological mechanisms underlying emotionally influenced memory now requires that we anticipate, and account for, the influence of sex (Shors 1998; Cahill et al. 2001) or gender (Cahill et al. 2004).

\section{MATERIALS AND METHODS}

\section{Subjects}

Twenty-three right-handed healthy volunteers (12 male; 11 female) participated in this study. The average age of the male subjects was $26.0 \pm 5.4$ years, and the average age of the female subjects was $23.6 \pm 3.5$ years. Subjects had no history of head injury, mental illness, or substance abuse, or had any counter indications for MRI. Subjects were recruited through posted advertisements and paid 40 dollars for their participation. All subjects were treated in accordance with the approved procedures of the institutional review boards of both the Veterans Affairs Medical Center in Long Beach, California, and the University of California-Irvine. A total of eight subjects were removed for the imaging analysis: four men and four women. Two of the women were removed for signal dropout in the medial temporal lobes, and the rest (four men, two women) were removed for movement $>3$ $\mathrm{mm}$, leaving a total of 15 subjects (eight men and seven women) in the imaging analysis.

\section{Behavioral Procedures}

The stimuli were those described by Canli et al. (2000), with some modifications in the manner in which they were presented. Subjects viewed 96 scenes from the International Affective Picture System (IAPS) stimuli set. The normative valence ratings for this set ranged from highly negative (1.17) to neutral (5.44), and the normative arousal ratings ranged from tranquil (1.97) to highly arousing (7.63). Further details regarding this set can be found in Canli et al. (2000). In the present experiment, the 96 scenes were presented in three blocks of 32 scenes matched in terms of normative arousal ratings. The order of the blocks was counterbalanced across subjects, and within each block, the order of scenes was randomized across subjects. Each scene was presented for 2.88 sec with an interstimulus interval of $12.96 \mathrm{sec}$, during which subjects viewed a fixation cross. Subjects were asked to indicate their emotional arousal after the scene disappeared by pressing a lever with their right hand. Subjects chose from four levers, indicating emotional arousal on a scale of one ("not emotionally arousing") to four ("highly emotionally arousing"). The subject's responses were recorded by the experimenter.

Two weeks after the scan, subjects received an unexpected recognition test in a room next to the scanner. During the recognition test, subjects viewed all of the previously seen pictures and 48 new IAPS scenes (foils) on a Dell Inspiron 8100. The foils were selected to match the previously seen pictures in their normative valence and arousal ratings (for details regarding this set, see Canli et al. 2000). Subjects were asked whether they had seen the picture during the scan 2 weeks earlier. When the subject judged a picture as previously seen, they were then asked to indicate whether they remembered with certainty ("remember") or had a less certain feeling of familiarity ("know"). Subjects also completed the Mehrabian scale designed to assess trait arousability (Mehrabian 1977), although these data were not included in the analyses.

\section{Scanning Procedures}

Data were acquired at the Long Beach Veterans Affairs Medical Center in a $1.5 \mathrm{~T}$ scanner manufactured by Marconi Medical Systems, Inc., with multislice echo-planar imaging capabilities developed and installed by Marconi. This system was used to acquire both T1-weighted anatomical volume images and T2*- 


\begin{tabular}{|c|c|c|c|c|}
\hline Brain region & Hemisphere & $\begin{array}{c}\text { Spatial } \\
\text { extent } \\
\text { (\# voxels) }\end{array}$ & $\begin{array}{c}\text { Talairach } \\
\text { coordinates } \\
(x, y, z)\end{array}$ & Z score \\
\hline \multicolumn{5}{|l|}{ Men: } \\
\hline Lateral parietal (BA 2) & $\mathrm{R}$ & 36 & $66,-22,29$ & 3.64 \\
\hline Globus pallidus & $\mathrm{R}$ & 8 & $18,-12,1$ & 3.69 \\
\hline Lateral parietal (BA 43) & $\mathrm{L}$ & 7 & $-56,-13,14$ & 3.86 \\
\hline Anterior hippocampus & $\mathrm{R}$ & 6 & $38,7,-22$ & 3.56 \\
\hline Posterior frontal (BA 6) & $\mathrm{R}$ & 6 & $52,-6,39$ & 3.41 \\
\hline Cerebellum & L & 4 & $-16,-40,-11$ & 3.36 \\
\hline \multicolumn{5}{|l|}{ Women: } \\
\hline Middle temporal gyrus & $\mathrm{L}$ & 15 & $-54,-39,-8$ & 4.21 \\
\hline Mid brainstem & $\mathrm{L}$ & 15 & $-2,-26,-10$ & 3.97 \\
\hline Posterior cingulate & L & 7 & $-10,-50,45$ & 4.05 \\
\hline Inferior parietal (BA40) & $\mathrm{L}$ & 4 & $-55,-40,28$ & 3.66 \\
\hline
\end{tabular}

Note: Inferences were made at $P<0.001$ (uncorrected for multiple comparisons) at the random-effects level with an extent threshold of 4 or more contiguous voxels. Z-score indicates maximal $Z$ score in the cluster reported.

less than one-eighth of this mean, (3) calculating a new voxel mean (termed the global mean), and (4) including in the analysis only those voxels with signal $>0.8$ of the global mean.

A voxelwise application of the general linear model to the time series images determined parameter estimates and variance for each covariate in a subject-specific fixed effects model. Intersubject variance was modeled in a random effects (second level) model (Friston et al. 1999). The variance in BOLD signal for each subject was decomposed into a set of regressors associated with arousal-related activity and subsequent memory performance-related activity. We identified regions showing emotional arousal-related modulation of memory-related activity by decomposing the variance in BOLD signal for each subject into event-related regressors and corresponding parametric modulatory regressors. To do so, stimuli were classified into three event-types of interest: high confidence hits, low confidence hits, and misses. The magnitude of the BOLD response to each event was modeled by convolving a $\delta$ function at each event onset with a canonical hemodynamic response function. An additional regressor for each event-type of corresponding emo-

weighted functional images with BOLD contrast. For each session, the magnetic gradient was automatically shimmed by using a first-order algorithm with the subject's head centered in the magnetic field. A high-resolution full-brain anatomical image was acquired for each subject at the beginning of each session.

Anatomical images were acquired by using a Fourieracquired steady-state technique (FAST) sequence with an inplane resolution of $0.94 \times 0.94 \mathrm{~mm}^{2}$ and a slice thickness of 2.5 $\mathrm{mm}$. Functional images were acquired by using a gradient-echo echo-planar imaging sequence $(\mathrm{TR}=1.807 \mathrm{sec}, \mathrm{TE}=40 \mathrm{msec}$, flip angle $=90$ degrees, fat-saturating prepulse). Each echo planar image consisted of sixteen $7-\mathrm{mm}$ axial slices of in-plane voxel size $1.88 \times 1.88 \mathrm{~mm}^{2}$. Three runs of 289 volumes each were collected continuously. The first three volumes in each run were discarded to account for T1 equilibration effects.

Stimuli were presented via MRI-compatible Silent Vision goggles. Silent Scan headphones from Avotec were used to communicate with participants in the scanner. While they were in the scanner, subjects wore earplugs and headphones. Head motion was reduced by lining the head coil with pads, taping subjects heads to the head coil, and instructing subjects to lie as still as possible in particular during actual scanning sequences.

\section{Data Analysis}

Image processing and statistical analyses were carried out by using the Statistical Parametric Mapping (SPM99) analysis package (http://www.fil.ion.ucl.ac.uk/spm) and MATLAB software (The MathWorks). Following standard functional image processing and analysis procedures, all volumes from each subject were realigned by using the first volume as a reference, and resliced by using sinc interpolation. The functional images were coregistered to the corresponding anatomical (T1-weighted) image, spatially normalized into standard stereotactic space (Friston et al. 1995) with respect to the MNI-305 template (Montreal Neurological Institute) applying nonlinear basis functions, and spatially smoothed by using an 8-mm FWHM isotropic Gaussian kernel. The time-series images were then high-pass filtered by using a discrete set of nonlinear basis functions with a cutoff period of $1 / 120 \mathrm{~Hz}$ to eliminate low-frequency periodicities, and scaled within-session to a grand mean of 100 . For temporal smoothing, the covariates were convolved with a canonical hemodynamic response function. Those subjects whose movement exceeded 3 $\mathrm{mm}(\mathrm{n}=5)$ were omitted from the analysis. In addition, to ensure that sufficient signal was obtained from all relevant amygdala voxels, a standard global mask (grey matter threshold) was applied to all voxels. This was done in SPM99 by (1) calculating the mean intensity of all voxels, (2) discarding any voxels with tional arousal ratings was used to model the parametric modulation of subsequent recognition performance by emotional arousal rating using a linear function. The contrast of the difference in modulatory regressors across memory performance levels identified regions where greater BOLD signal was associated with both better memory performance and increasing arousal ratings. Given our hypothesis regarding the effect of arousal and subsequent memory within the amygdala, a region of interest analysis was performed with small volume correction for multiple comparisons (sphere radius $=5 \mathrm{~mm}$ ), centering on those voxels in the amygdala that showed significant activations in the specified model (Worsley et al. 1996).

To provide additional documentation of a sex-related hemispheric lateralization of amygdala function in relation to memory for arousing stimuli, a sex-by-hemisphere ANOVA was performed. To obtain parameter estimates for the height of the BOLD response for men and women, each group was analyzed independently with the contrast described above (the parametric modulation of memory by arousal) in a random-effects analysis. The parameter estimates of the independent group analyses were adjusted for confounds and fitted to the group grand mean. The adjusted parameter estimates from each group's maximally activated voxel within each subject's amygdala were used to analyze interactions between sex and hemisphere. A liberal statistical threshold $(P<0.3)$ was needed to obtain parameter estimates from the homotopic voxel in the amygdala of opposing hemisphere (left in men, right in women). Thus, two values were obtained for each subject: the $\beta$ at the peak voxel and the $\beta$ for the homotopic voxel in the opposite hemisphere. These betas constituted the dependent variables in a $2 \times 2$ repeated-measures ANOVA. To avoid bias because parameter estimates were selected based on activations from the above contrast, a second ANOVA was performed using amygdala voxel coordinates from a previous study (Canli et al, 2002). Behavioral data concerning arousal ratings and memory were investigated by using ANOVA and unpaired $t$-tests.

\section{ACKNOWLEDGMENTS}

Supported by National Institute of Mental Health grant $\mathrm{MH}$ 57508 and a Desert Pacific Mental Illness Research, Education and Clinical Centers (MIRECC) Award to L.C.

The publication costs of this article were defrayed in part by payment of page charges. This article must therefore be hereby marked "advertisement" in accordance with 18 USC section 1734 solely to indicate this fact. 


\section{REFERENCES}

Adinoff, B., Devous, M., Best, S., Chandler, P., Alexander, D., Payne, K., Harris, T., and Williams M. 2003. Gender differences in limbic responsiveness, by SPECT, following a pharmacologic challenge in healthy subjects. Neuroimage 18: 697-706.

Buchanan, T., Denburg, N., Tranel, D., and Adolphs, R. 2001. Verbal and nonverbal emotional memory following unilateral amygdala damage. Learn. Mem. 8: 326-335.

Cahill, L. 2000. Emotional modulation of long-term memory storage in humans: Adrenergic activation and the amygdala. In: The amygdala: A functional analysis (ed. J. Aggleton), pp. 425-444. Oxford University Press, Oxford.

Cahill, L. and McGaugh, J.L. 1990. Amygdaloid complex lesions differentially affect retention of tasks using appetitive and aversive reinforcement. Behav. Neurosci. 104: 532-543.

Cahill, L. and van Stegeren, A. 2003. Sex-related impairment of memory for emotional events with $\beta$-adrenergic blockade. Neurobiol. Learn. Mem. 79: 81-88.

Cahill, L. Haier, R., Fallon, J., Alkire, M., Tang, C., Keator, D., Wu, J., and McGaugh, J.L. 1996. Amygdala activity at encoding correlated with long-term, free recall of emotional information. Proc. Natl. Acad. Sci. 93: 8016-8021.

Cahill, L., Haier, R.J., White, N.S., Fallon, J., Kilpatrick, L., Lawrence, C., Potkin, S.G., and Alkire, M.T. 2001. Sex-related difference in amygdala activity during emotionally influenced memory storage. Neurobiol. Learn. Mem. 75: 1-9.

Cahill, L., Gorski, L., Belcher, A., and Huynh, Q. 2004. A study of the influence of sex versus sex-related traits on long-term recall of gist and detail from an emotional story. Consciousness and Cognition (in press).

Canli, T., Zhao, Z., Desmond, J., Glover, G., and Gabrieli, J. 1999. fMRI identifies a network of structures correlated with retention of positive and negative emotional memory. Psychobiology 27: 441-452.

Canli, T., Zhao, Z., Brewer, J., Gabrieli, J.D., and Cahill, L. 2000. Event-related activation in the human amygdala associates with later memory for individual emotional experience. J. Neurosci. 20: RC99.

Canli, T., Zhao, Z., Desmond, J., Zhao, Z., and Gabrieli, J. 2002. Sex differences in the neural basis of emotional memories. Proc. Natl. Acad. Sci. 99: 10789-10794.

Delis, D.C., Robertson, L.C., and Efron, R. 1986. Hemispheric specialization of memory for visual hierarchical stimuli. Neuropsychologia 24: 205-214.

Durston, S., Hulshoff Pol, H.E., Casey, B.J., Giedd, J.N., Buitelaar, J.K., van Engeland, H. 2001. Anatomical MRI of the developing human brain: What have we learned? J. Am. Acad. Child Adolesc. Psychiatry. 40: 1012-1020.

Fink, G.R., Halligan, P.W., Marshall, J.C., Frith, C.D., Frackowiak, R.S.J., and Dolan, R.J. 1997. Neural mechanisms involved in the processing of global and local aspects of hierarchically organized visual stimuli. Brain 120: 1779-1791.

Friston, K., Holmes, A., Worsely, K., Poline, J., Frith, C., and Frackowiak, R. 1995. Statistical parametric maps in functional imaging: A genera linear approach. Hum. Brain Mapp. 2: 189-210.

Friston, K., Holmes, A., Price, C.J., Buchel, C., and Worsley, K. 1999. Multisubject fMRI studies and conjunction analyses. Neuroimage 10: $385-396$.
Giedd, J., Castellanos, F., Rajapakse, J., Vaituzis, A., and Rapoport, J. 1997. Sexual dimorphism of the developing human brain. Prog. Neuropsychopharmacol. Biol. Psychiatry 21: 1185-1201.

Goldstein, J., Seidman, L.J., Horton, N.J., Makris, N., Kennedy, D.N., Caviness Jr., V.S., Faraone, S.V., and Tsuang, M.T. 2001. Normal sexual dimorphism of the adult human brain assessed by in vivo magnetic resonance imaging. Cerebral Cortex 11: 490-497.

Gur, R.C., Gunning-Dixon, F., Bilker, W.B., Gur, R.E. 2002. Sex differences in temporo-limbic and frontal brain volumes of healthy adults. Cereb. Cortex. 12: 998-1003.

Hamann, S., Ely, T., Grafton, S., and Kilts, C. 1999. Amygdala activity related to enhanced memory for pleasant and aversive stimuli. Nat. Neurosci. 2: 289-293.

Ivry, R.B. and Robertson, L.C. 1998. The two sides of perception. Bradford Book, London.

Killgore, W.D.S., Oki, M., and Yurgelun-Todd, D.A. 2001. Sex-specific developmental changes in amygdala responses to affective faces. NeuroReport 12: 427-433.

Kilpatrick, L. and Cahill, L. 2003. Amygdala modulation of parahippocampal and frontal regions during emotionally influenced memory storage. Neuroimage 20: 2091-2099.

LaBar, K., Parrish, T., Gitelman, D., and Mesulam, M.-M. 2001. Impact of signal-to-noise on functional MRI of the amygdala during emotional picture encoding. Neuroreport 12: 3461-3464.

McGaugh, J.L. 2000. Memory: A century of consolidation. Science 287: 248-251.

Mehrabian, A. 1977. Individual differences in stimulus screening and arousability. J. Pers. 45: 237-250.

Morris, J.S., Ohman, A., and Dolan, R.J. 1998. Conscious and unconscious emotional learning in the human amygdala. Nature 393: $467-470$.

Phelps, E., O'Connor, K., Gatenby, J., Gore, J., Grillon, C., and Davis, M. 2001. Activation of the left amygdala to a cognitive representation of fear. Nat. Neurosci. 4: 437-441.

Pizzagalli, D., Shackman, A., and Davidson, R.J. 2003. The functional neuroimaging of human emotion: Asymmetric contributions of cortical and subcortical circuitry. In: The asymmetrical brain (eds. R.J. Davidson and K. Hugdahl), p. 528. MIT Press, Cambridge, MA.

Shors, T.J. 1998. Stress and sex effects on associative learning: For better or for worse. Neuroscientist 4: 353-364.

Toufexis D.J. and Davis M. 2002. Involvement of progesterone in the reduction of CRF-enhanced startle during lactation in the rat. 32nd Annual Meeting of The Society for Neuroscience, Orlando FL. November 2-7.

Wager, T.D., Phan, K.L., Liberzon, I., and Taylor, S.T. 2003. Valence, gender, and lateralization of functional brain anatomy in emotion: A meta-analysis of findings from neuroimaging. Neuroimage 19: 513-531.

Worsley, K.J., Marrett, P., Neelin, A.C., Friston, K.J., and Evans, A.C. 1996. A unified statistical approach for determining significant signals in images of cerebral activation. Hum. Brain Mapp. 4: 58-73.

Zubieta, J-K., Dannals, R., and Frost, J. 1999. Gender and age influences on human brain $\mu$-opioid receptor binding measured by PET. Am. J. Psych. 156: 842-848.

Received September 11, 2003; accepted in revised form March 12, 2004. 


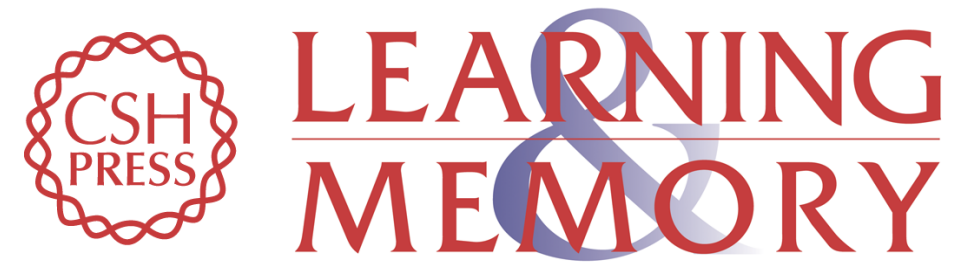

\section{Sex-Related Hemispheric Lateralization of Amygdala Function in Emotionally Influenced Memory: An fMRI Investigation}

Larry Cahill, Melina Uncapher, Lisa Kilpatrick, et al.

Learn. Mem. 2004, 11:

Access the most recent version at doi:10.1101/lm.70504

References This article cites 28 articles, 4 of which can be accessed free at: http://learnmem.cshlp.org/content/11/3/261.full.html\#ref-list-1

License

Email Alerting Receive free email alerts when new articles cite this article - sign up in the box at the Service top right corner of the article or click here. 